\title{
Exosomes promote pre-metastatic niche formation in ovarian cancer
}

\author{
Wenlong Feng ${ }^{1,2}$, Dylan C. Dean ${ }^{2}$, Francis J. Hornicek ${ }^{2}$, Huirong Shi ${ }^{{ }^{*}}$ and Zhenfeng Duan ${ }^{1,2^{*}}$ (D)
}

\begin{abstract}
Ovarian cancer is one of the most common gynecological malignancies. Upon initial diagnosis, the majority of patients present with widespread metastatic growth within the peritoneal cavity. This metastatic growth occurs in stages, with the formation of a pre-metastatic niche occurring prior to macroscopic tumor cell invasion. Exosomes released by the primary ovarian tumor are small extracellular vesicles which prepare the distant tumor microenvironment for accelerated metastatic invasion. They regulate intercellular communication between tumor cells and normal stroma, cancer-associated fibroblasts, and local immune cells within the tumor microenvironment. In this review, we highlight the emerging roles of ovarian cancer exosomes as coordinators of pre-metastatic niche formation, biomarkers amenable to liquid biopsy, and targets of chemotherapy.
\end{abstract}

Keywords: Ovarian cancer, Exosome, Pre-metastatic niche, Metastasis

\section{Highlights}

- Ovarian cancer is the deadliest gynecological malignancy, largely stemming from peritoneal metastasis. Formation of the pre-metastatic niche supports subsequent metastatic lesions.

- Ovarian cancer derived exosomes induce premetastatic niche formation via immunosuppression, angiogenesis, stromal cell remodeling, and oncogenic reprogramming.

- Ovarian cancer derived exosomes are promising biomarkers and therapeutic targets.

\section{Background}

Ovarian cancer is the most lethal gynecological malignancy, accounting for $2.5 \%$ of all female cancers and $5 \%$ of female cancer-related deaths [1]. In the United States, there were approximately 22,240 new cases of ovarian cancer diagnosed and 14,070 deaths in 2018 [2]. Ovarian cancer patients experience high mortality rates due to commonly being diagnosed during the late stage (III or IV) with bowel obstruction and systemic involvement [3]. The 5-year survival rate of ovarian cancer among

\footnotetext{
* Correspondence: fccshihr@zzu.edu.cn; zduan@mednet.ucla.edu ${ }^{1}$ Department of Obstetrics and Gynecology, The First Affiliated Hospital of Zhengzhou University, 1 Jianshe East Road, Zhengzhou 450052, Henan, China

Full list of author information is available at the end of the article
}

these late-stage III or IV cases is less than $29 \%$, as compared to $70 \%$ in early stage I cases [4]. There is, therefore, an urgent need to characterize the mechanisms of ovarian cancer metastasis and associated biomarkers in order to earlier diagnose and treat ovarian cancer patients within the stage I period.

Recent works have shown the pre-metastatic niche in ovarian cancer to be a prevalent precondition of metastasis [5]. The pre-metastatic niche is a preformed microenvironment made possible by exosomes secreted by the primary tumor site prior to widespread metastasis [6-8]. These exosomes optimize the environment for ovarian cancer colonization, outgrowth, and metastasis [9, 10]. This environment is mediated through immune suppression and evasion, angiogenesis, cancer-associated fibroblasts (CAF), and tumor macrophages that remodel the local stroma. Various studies have shown exosomes to play critical roles in tumorigenesis, growth, apoptosis, immune response, and chemotherapeutic resistance in cancer [11-15] (Fig. 1a). However, it was not until very recently that their roles in pre-metastatic niche establishment have been appreciated $[15,16]$. Despite their diameter of $30-100 \mathrm{~nm}$, these disk-shaped membranous vesicles contain unique signatures of proteins, lipids, DNA, and RNA making them powerful tumorigenic factors and diagnostic biomarkers [17]. In ovarian cancer, exosomes are detectable in both ascites and blood, 


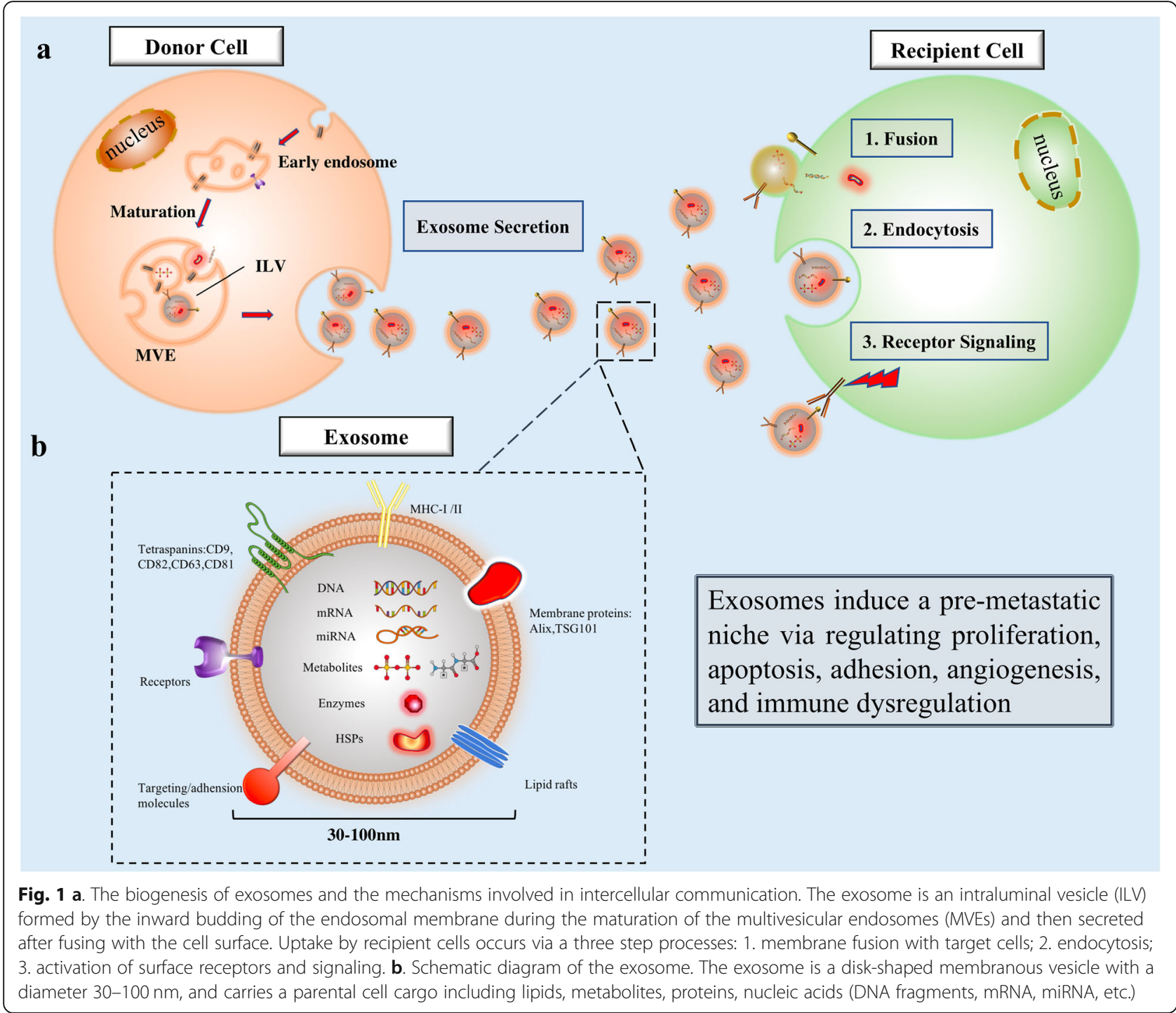

making them amenable to less invasive diagnostics and a potential target of earlier targeted therapy [18-20]. In this review, we summarize the functions of exosomes in ovarian cancer metastasis with respect to pre-metastatic niche formation.

\section{Exosome biogenesis}

The nature and abundance of exosomes are dictated by their donor cell of origin - whether it be healthy tissue or tumor [21]. Exosome biogenesis involves a set sequence of cellular events which eventually render a carcinogenic or physiologic subtype. The donor cells first internalize extracellular ligands and products to form early endosomes. Nascent exosomes then arise as intraluminal vesicles (ILVs) within the lumen of these endosomes, eventually maturing into multivesicular endosomes (MVEs) via selective inclusion of proteins, nucleic acids, and lipids. Whereas some MVEs fuse and degrade within lysosomes to provide the necessary energy and materials for cell formation, others are released into the extracellular environment through Golgi recirculation or direct cell secretion [22]. The endosomal sorting complex required for transport (ESCRT) is a primary driver of membrane shaping and scission, and the first accepted mechanism of MVE and ILV formation [23]. The final exosomal cargo drives its eventual function, and is achieved by a highly selective series of regulatory stages. It involves endoprotein sorting and precise ESCT cleavage of ubiquitinated proteins into ILVs via ESCT-0, -I, -II, and -III as well as VPS4, VTA1, ALIX/PDCD6IP, and TSG101. Lipids such as ceramide, cholesterol, and the four-transmembrane protein superfamily including CD9, CD63, and CD81 also mediate exosome protein sorting. At the nucleic acid level, exosomal mRNAs are sorted into MVEs according to the Z-zipper structure of their 3'UTR as well as through RNA-induced silencing complexes (RISCs) [24, 25]. An ARF6-Exportin-5 axis also 
delivers pre-miRNA cargo into tumor microvesicles [26]. These regulatory methods demonstrate the mechanism by which ovarian cancer exosomes obtain their distinct molecular signature from surrounding healthy tissue.

\section{Ovarian cancer-exosomes}

Ovarian cancer is distinguished from other human tumors by its preference for peritoneal cavity invasion through the ascites, making it especially adept at involving various viscera within the compartment. From the early stages on, the ascites contains detached tumor cells, various immune cells, mesothelial cells, and tumor associated exosomes. These exosomes can be isolated from the ascites [27, 28] and serum $[29,30]$ of patients with ovarian cancer. Importantly, these exosomes contain unique protein signatures specific to ovarian cancer, including membrane proteins (Alix, TSG 101), small GTPases (Rab proteins), annexin proteins, tetraspanins (CD9, CD82, CD63 and CD81), heat shock proteins (Hsp90, Hsc70), antigens (MHC I and II), Nanog and enzymes (phosphate isomerase, peroxiredoxin, aldehyde reductase, fatty acid synthase) (Fig. 1b). In addition to revealing an underlying malignancy, the exosomal protein cargo functions to enhance the progression of metastasis of ovarian tumors (Table 1). For example, Nanog is a transcription regulator involved in tumor cell proliferation and self-renewal of cancer stem cells [31]. Nanog expression is significantly greater in exosomes sampled from the ascites of high-grade serous ovarian cancer compared to benign peritoneal fluid [32]. Nanog knockout studies have shown decreased migration and invasion of ovarian cancer cells [33].

Tumorigenic microRNAs (miRNA) have also been identified within exosomes, where they are encapsulated and protected from nuclease degradation. Functionally, these intra-exosomal miRNAs regulate gene expression of target cells both locally and systemically. Once shed, the ovarian cancer derived exosomes horizontally transport their miRNAs to tumor-associated macrophages (TAMs), mesothelial cells, and tumor cells themselves. They provide instructions for pre-metastatic niche formation and metastasis in the nascent stages of malignancy (Table 2).

\section{Exosomes cause immunosuppression within the pre- metastatic niche}

The immune system is a significant barrier to metastasis. For ovarian cancer to thrive in its new environment, it is therefore principally important for the premetastatic niche to protect the metastatic cells from being apoptosed upon entering the metastatic site. Exosomes isolated from the ascites of ovarian cancer can induce a rapid and reversible $\mathrm{T}$ cell arrest [17]. One recent study found GD3, a ganglioside expressed on the surface of exosomes isolated from ascites, to arrest $\mathrm{T}$ cells via acting on their T-cell receptor (TCR) [46]. Ovarian cancer associated exosomes can also induce the production of IL-6 within monocytes through toll-like receptor (TLR) activation. IL- 6 then activates the signal transducer and activator of transcription 3 (STAT3) pathway in immune cells, stromal cells, and tumor cells, which supports overall immune escape of cancer cells [47] (Fig. 2a).

With the aim of revealing the direct effect of exosomes on immune cells, various works have cocultured exosomes from the ascites of ovarian cancer patients with dendritic cells, hematopoietic stem cells, and peripheral blood lymphocytes. One such study showed exosomes to cause immunosuppression by inducing apoptosis of hematopoietic stem cells, dendritic cells, and peripheral blood lymphocytes [27] (Fig. 2a). In another study, normal peripheral blood lymphocytes were cocultured with exosomes from ovarian cancer ascites or peritoneal washings of patients with benign ovarian cysts with follow up gene expression analysis of the lymphocytes. They found overexpression of 26 immunosuppressive genes in the malignant ascites cocultured group compared to the benign ovarian cyst group. These findings support the role of exosomes in dampening peripheral immunity through direct interaction with leukocytes, allowing for unmitigated tumor invasion [48].

Both the adaptive immune response, including CD4+ and CD8+ T cells, as well as innate immune cells such

Table 1 Summary of ovarian cancer exosomal proteins involved with metastasis

\begin{tabular}{llll}
\hline Exosomal Proteins & Recipient cells & Role/Mechanism & Reference \\
\hline Proteomic analysis: 2230 proteins were identified & N/A & Tumorigenesis and metastasis & [34] \\
ATF2, MTA1, ROCK1/2 & HUVECS & Angiogenesis & [35] \\
GNA12, EPHA2 and COIA1 & MSCs and ECs & Promote MSC and EC migration for metastasis \\
CD44 & HPMCs & Tumor cell invasion. & [36] \\
RNA-binding protein LIN28 & HEK293 cells & Increase HEK293 cell invasion and migration & [38] \\
Nanog & N/A & Tumor cell proliferation and invasion & [32, 33]
\end{tabular}

Abbreviations: ATF2 Activating transcription factor 2, MTA1 Metastasis-associated protein 1, ROCK Rho-Associated, Coiled-Coil Containing Protein Kinase, HUVECS Human umbilical vein endothelial cells, GNA12 Guanine nucleotide-binding protein subunit alpha-12, EPHA2 ephrin type-A receptor 2, COIA1 Collagen alpha-1 (XVIII) chain, MSCs Mesenchymal stem cells, ECs endothelial cells, HPMCs Human peritoneal mesothelial cells, LIN28 Lin-28 homolog A, HEK293 human embryonic kidney 
Table 2 Summary of ovarian cancer exosomal miRNAs involved with metastasis

\begin{tabular}{llll}
\hline Exosomal miRNAs & Recipient cells & Role/Mechanism & Reference \\
\hline $\begin{array}{l}\text { miR-940, miR-222-3p,miR-21-3p, } \\
\text { miR-125 b-5p, miR-181 d-5p }\end{array}$ & TAMs & M2 phenotype polarization, EOC proliferation and migration \\
miRNA 21 and 29a & ES2 OC cellsLP9 mesothelial cells & Mesothelial cell clearance & [391] \\
miR-99a-5p & HPMC & Cell invasion through fibronectin and vitronectin upregulation & [43] \\
MMP1 mRNAs & MeT-5A and HPMC & Destruction of peritoneal mesothelium barrier & [44] \\
let-7a-f and miR-200a-c & N/A & Correlates with ovarian cancer invasiveness & [45] \\
\hline
\end{tabular}

Abbreviations: TAMs Tumor-associated macrophages, HPMC Human peritoneal mesothelial cells, OC ovarian cancer, EOC Epithelial ovarian cancer, MMP Matrix metallopeptidase

as natural killer (NK) cells and macrophages, are strongly recruited to the metastatic site. These immune cells are potent physiologic defenses against tumor progression [49]. A growing body of evidence suggests exosomes derived from ovarian cancer cells can silence these immune cells in the tumor microenvironment and are critical in pre-metastatic niche formation [50-52]. In addition to reprogramming the immune cell gene profile, ovarian cancer cells release Fas ligand (FasL)-carrying exosomes which downregulate the expression of the

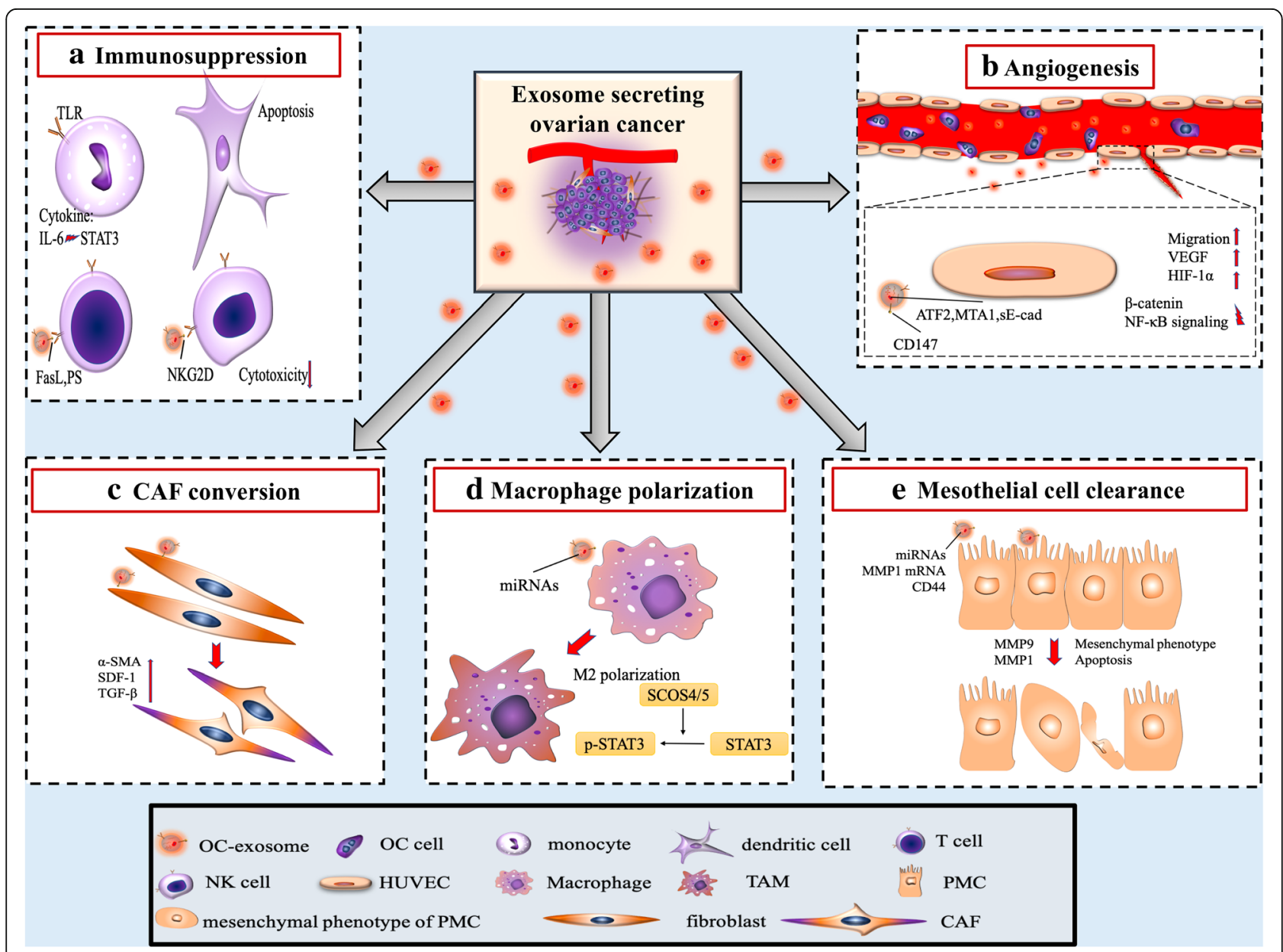

Fig. 2 Impact of ovarian cancer-exosomes on target cells during pre-metastatic niche formation. a. Ovarian cancer-exosomes induce apoptosis of dendritic cells, increase secretion of IL-6, and inhibit function of T cells and NK cells. b. Ovarian cancer-exosomes with their cargo, such as ATF2, MTA1, SE-cad, and CD147, which acts on human vein endothelial cells, inducing angiogenesis and vascular permeability. c. Ovarian cancerexosomes convert fibroblasts to CAFs. $\mathbf{d}$. Ovarian cancer-exosomes deliver miRNAs to macrophages and elicit M2 macrophage polarization by regulating the suppressor of cytokine signaling (SOCS)4/5/ STAT3 pathway in macrophages. e. Phenotypic conversion and apoptosis of PMCs, induced by ovarian cancer-exosomes, clears the mesothelial cell barrier of the peritoneum and omentum. OC ovarian cancer, NK natural killer, ATF2 Activating transcription factor 2, MTA1 Metastasis-associated protein 1, sE-cad soluble E-cadherin, SOCS Suppress cytokine signaling, STAT3 Signal transducer and activator of transcription 3, PMC Peritoneal mesothelial cell, CAF cancer-associated fibroblast 
surface T-cell receptor/CD3- zeta $(\zeta)$ and promote T-cell apoptosis [53, 54]. At the cellular level, lysophosphatidic acid elevates the expression of FasL on the surface of ovarian cancer cells, thus prompting the release of FasLcarrying exosomes [55]. In addition, ovarian cancer exosomes carry phosphatidylserine, which inhibits T-cell activation by blocking intracellular signaling cascades [56]. NK cells are prominent innate immune effector cells that recognize and kill tumor cells directly. The NKG2D receptor-ligand is a crucial activating cytotoxic receptor of NK cells and a target of ovarian cancer exosome downregulation. Inhibition of NKG2D circumvents NK cell cytotoxicity, allowing for immune evasion of tumor cells and a robust pre-metastatic niche [57] (Table 3) (Fig. 2a).

Exosomes promote angiogenesis in the pre-metastatic niche To ensure adequate blood supply, VEGFR $1^{+}$hematopoietic progenitor cells initiate angiogenesis within the premetastatic niche [9]. Additionally, local endothelial progenitor cells promote angiogenesis via VEGF signaling [52]. Aside from blood vessel growth, the proangiogenic microenvironment increases vascular permeability towards the pre-metastatic niche for multiple cell types, including VEGFR1+ hematopoietic progenitor cells, immune cells, stromal cells, and the homing of tumor cells [52]. This antecedent angiogenesis makes the pre-metastatic niche capable of meeting the nutrient requirements for subsequent rapid metastatic growth. Ovarian cancer exosomes have recently gained notoriety for promoting angiogenesis (Fig. 2b). One such study demonstrated their ability to enhance viability and migration of human umbilical vein endothelial cells. At the molecular level, proteomics have revealed activating transcription factor 2 (ATF2) and metastasis-associated protein 1 (MTA1) housed within ovarian cancer exosomes to upregulate angiogenesis [58]. CD147 is a tumorigenic membrane-bound molecule expressed in cancer cells which regulates matrix metalloproteinase expression in peritumoral stromal cells. CD147positive exosomes released by ovarian tumors promote angiogenesis in human umbilical vein endothelial cells as well [59]. In a uniquely VEGF-independent manner, soluble E-cadherin (sE-cad) harboring exosomes are present within the ascites of ovarian cancer patients and are robust activators of angiogenesis. Mechanistically, sE-cad-positive exosomes bind with VE-cadherin on endothelial cells, prompting a signaling cascade that ultimately activates $\beta$ catenin and NF-kB; this stimulates endothelial cell migration and overall vascular permeability [60]. Despite their small size, a growing body of research is supporting the role of ovarian cancer derived exosomes in cultivating an angiogenic tumor niche for widespread peritoneal metastasis.

\section{Exosomes in stromal remodeling}

The survival of cancer cells that metastasize from primary tumors to secondary sites depends upon the stroma microenvironment. Tumor derived exosomes assist in this process by educating and remodeling stromal cells in the metastatic site to support tumor cell viability and metastatic dissemination. Functionally, they reprogram stromal cells in the pre-metastatic niche including cancer-associated fibroblasts (CAFs) and pericytes. Exosomes also interact with tumor-associated macrophages (TAM) in the metastatic microenvironment, which are

Table 3 Roles of ovarian cancer exosomes in pre-metastatic niche formation

\begin{tabular}{|c|c|c|c|c|c|}
\hline Exosome Function & Origin of exosomes & Recipient cells & Effect & Potential targets & Ref. \\
\hline \multirow[t]{7}{*}{ Immunosuppression } & Ascites of OC patients & T cells & Arrest T cells & GD3, TCR & {$[46]$} \\
\hline & Ascites of OC patients & Monocytes & Cytokine production & TLR signaling pathways & {$[47]$} \\
\hline & Ascites of OC patients & PBMCs and DCs & Apoptosis & FasL & {$[27]$} \\
\hline & Ascites of OC patients & T cells & Apoptosis & FasL & {$[54]$} \\
\hline & OC cells & T cells & Counterattack against activated $T$ cells & LPA and FasL & {$[55]$} \\
\hline & Ovarian tumor tissues & T cells & T cell inhibition & Depletion or blockade of PS & {$[56]$} \\
\hline & EOC cells and ascites & NK cells & Cytotoxicity inhibition & NKG2D & {$[57]$} \\
\hline \multirow[t]{3}{*}{ Angiogenesis } & OC cells & HUVECS & Affect VEGF or HIF-1a & ATF2 and MTA1 & {$[58]$} \\
\hline & OC cells & HUVECS & CD147 stimulates VEGF expression & CD147 & {$[59]$} \\
\hline & OC cells & HUVECS & Activate $\beta$-catenin and NF-kB signaling & sE-cad & {$[60]$} \\
\hline \multirow[t]{2}{*}{ Stroma remodeling } & OC cells & ADSCs & Transition ADSCs to CAFs & $\begin{array}{l}\text { a-SMA, SDF-1, TGF- } \beta \text { SMAD2 and } \\
\text { PI3K AKT pathway }\end{array}$ & [67] \\
\hline & OC cells & Macrophages & Macrophage M2 polarization & $\begin{array}{l}\text { miR-21-3p, miR-125b-5p, and } \\
\text { miR-181d-5p SOCS4/5/ STAT3 pathway }\end{array}$ & {$[41]$} \\
\hline
\end{tabular}

Abbreviations: OC ovarian cancer, GD 3 ganglioside 3, TCR T-cell receptor, TLR Toll-like receptor, PBMCs peripheral blood lymphocytes, DCs dendritic cells, FasL Fas ligand, LPA Lysophosphatidic acid, PS phosphatidylserine, EOC Epithelial ovarian cancer, NK natural killer, HIF Hypoxia-inducible factor, HUVECs Human umbilical vein endothelial cells, $s E$-cad soluble E-cadherin, ADSCs adipose tissue-derived mesenchymal stem cells, $a$-SMA alpha-smooth muscle actin, SDF-1 stromal cellderived factor 1, PI3K Phosphoinositide 3-kinases, AKT Protein kinase B, SOCS Suppress cytokine signaling, STAT3 Signal transducer and activator of transcription 3 
responsible for tumor growth, invasion, angiogenesis, and overall metastasis.

\section{Exosomes convert fibroblasts to CAFs}

CAFs are unique, reprogrammed stromal cells with roles in cancer initiation, extracellular matrix remodeling, progression, pre-metastatic niche formation, and metastasis [61]. They secrete a tumorigenic cytokine milieu of TGF- $\beta$, stromal cell-derived factor- $1 \alpha$ (SDF- $1 \alpha)$, S100A4, fibronectin, and matrix metalloproteinases in the local stromal cell microenvironment [62, 63]. While these signaling molecules have unique and specific processes in forming the tumor microenvironment, they all contribute to stromal remodeling within the pre-metastatic niche $[62,64]$. In a study where the miRNA profile between normal fibroblasts and ovarian tumor-adjacent fibroblasts were compared, ovarian tumor-adjacent fibroblasts consistently showed miR-31 and miR-214 downregulation with miR-155 upregulation. These researchers demonstrated the significance of this miRNA signature by transfecting miR-31 and miR-214 mimics or miR-155 inhibitors into normal fibroblasts, which caused them to convert to CAFs [65]. This showed ovarian cancer derived exosomes alone are sufficient to induce the phenotypic and functional changes in normal stromal fibroblasts to pathogenic CAFs [66] (Fig. 2c). Similar work has supported the role of exosomes in transitioning normal stroma to CAFs. Ovarian cancer is able to transition adipose derived mesenchymal stem cells to CAFs by overexpressing alpha-smooth muscle actin ( $\alpha$-SMA), SDF- 1 and TGF- $\beta$ [67]. Reprogramming of normal stroma to cancerous stroma can therefore be mediated by cytokine signaling from exosomes prior to secondary metastatic growth.

\section{Exosomes induce macrophage polarization}

Macrophages are multifunctional antigen presenting cells classically categorized into two polarized phenotypes: pro-inflammatory (M1) and anti-inflammatory (M2) [68]. Tumor associate-macrophages (TAMs) are of the M2 subtype and permeate malignant tissues [69]. Within the tumor microenvironment, TAMs secrete IL4 , IL-5, and IL-6, which promote angiogenesis, matrix remodeling, and immune system suppression [70]. They also contribute to the pre-metastatic niche by secreting TGF- $\beta$, SDF- 1 , and VEGF via the STAT3 signaling cascade [71]. In ovarian cancer, hypoxia-inducible factors (HIFs) induce the release of exosomes enriched with various miRNAs, including miR-21-3p, miR-125 b-5p, and miR-181 d-5p. When these exosomes are phagocytosed by undifferentiated macrophages, they undergo M2 polarization via the suppressor of cytokine signaling (SOCS)4/5/ STAT3 pathway [41] (Fig. 2d). One recent study showed ovarian cancer cells co-cultured with macrophages are only capable of transferring their oncogenic miR-1246 via exosomes to M2 macrophages and not to M1 macrophages. As a follow up, a combination miR-1246 inhibitor and chemotherapy regimen significantly reduced tumor burden in vivo [72]. These emerging works have supported the role of directing the M2 subtype at multiple stages of tumorigenesis and pre-metastatic niche formation.

\section{Exosome-educated cells in the pre-metastatic niche promote metastasis}

Ovarian cancer exosomes directly promote circulating tumor cell homing, colonization, and outgrowth within the premetastatic niche while suppressing the host antitumor immune response. They also enable tumor cell proliferation and invasion by encouraging host cell transformation to TAM and CAF phenotypes (Table 3). Experimental evidence has shown exosome-educated TAMs to enhance ovarian cancer proliferation and migration in vitro and with in vivo mouse models [41]. This occurs as a result of the cytokine profile of TAMs, whereby they secrete large amounts of EGF which activate EGFR in peripheral ovarian cancer cells. This EGF/ EGFR signaling cascade upregulates vascular endothelial growth factor-C (VEGF-C), which in turn upregulates integrin and intercellular adhesion molecule (ICAM-1). This induces proliferation, migration, adhesion, spheroid formation, and peritoneal implantation of ovarian cancer cells [73] (Fig. 3b). In the pre-metastatic niche, the tumor derived exosomes convert local fibroblasts into CAFs, which support tumorigenesis through their own exosome secretion. When ovarian cancer cells ingest TGF $\beta 1$-enriched CAF-exosomes, they upregulate TGF $\beta 1$ expression and become more adept at migration and invasion via a SMAD signaling cascade [74]. Aside from this function, CAFs also enhance basement membrane permeability allowing tumor cells to better invade the local uninvolved stroma [75].

There is a unique interplay between chemotherapeutic and exosome function. Intriguingly, it was recently shown that breast cancer exosomes have enhanced pre-metastatic niche forming ability in the lung after a chemotherapy application [76]. In another study, researchers showed the first-line chemotherapeutic cisplatin to increase IL6-producing myofibroblastic CAFs in ovarian cancer patients by activating the $\mathrm{NFKB}$ signaling pathway [77]. Furthermore, they demonstrated pretreatment with the well-known diabetes drug metformin arrested stromal $\mathrm{NF} \kappa \mathrm{B} / \mathrm{IL} 6$ activation and lessened the chemoresistance response in ovarian cancer cells [77]. Metastatic and drug-resistant, recurrent ovarian cancer produces significantly higher IL-6 compared to matched primary tumors. When elevated, IL- 6 within the ascites of ovarian cancer patients correlates with poor outcomes [78-81]. 


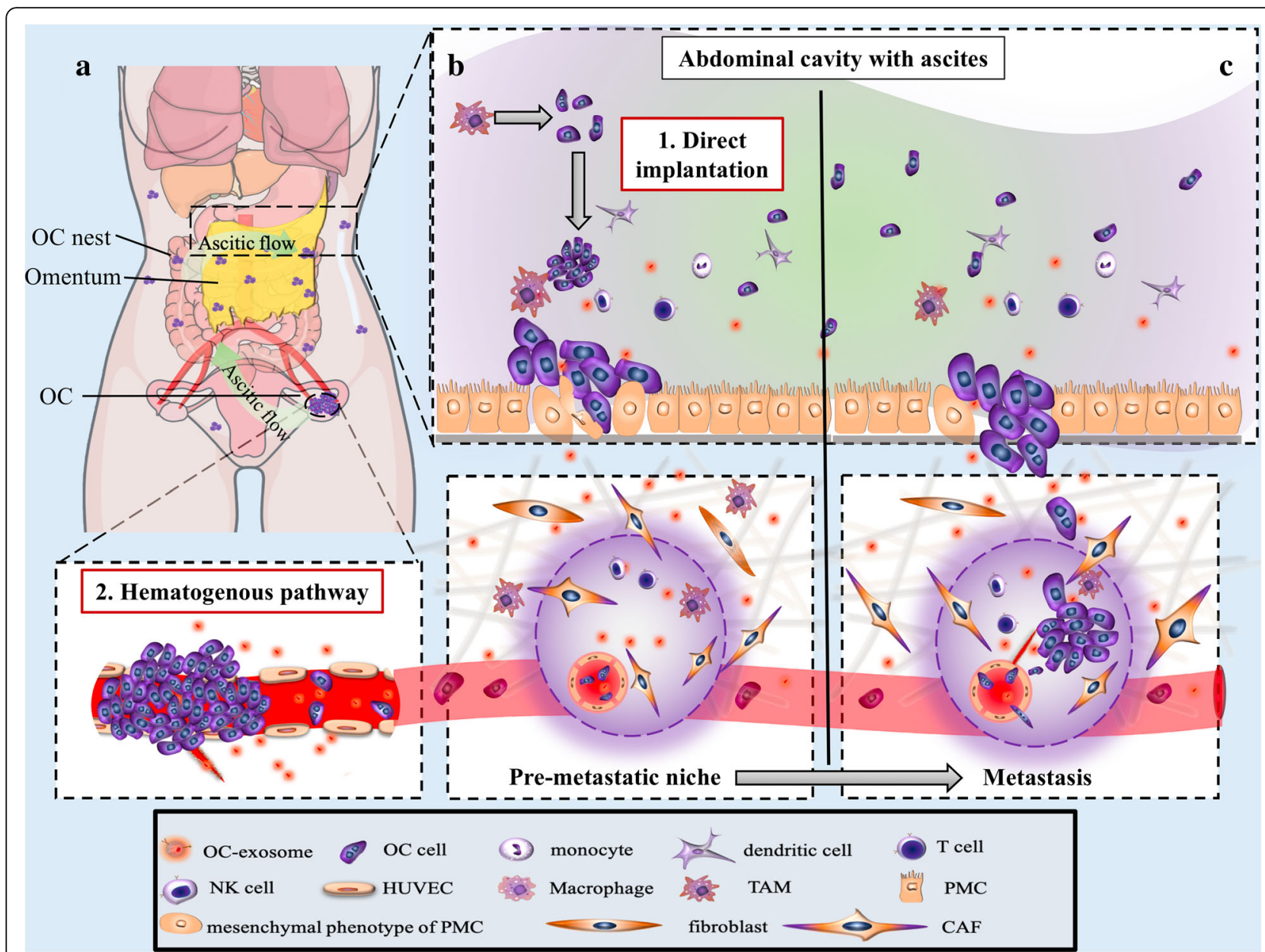

Fig. 3 Two main methods of ovarian cancer metastasis within the peritoneal cavity, direct implantation and hematogenous spread. a Distribution of ovarian cancer nests in the peritoneal cavity. $\mathbf{b}$ Mechanisms of ovarian cancer-exosome establishment of the pre-metastatic niche before metastasis. c After the formation of pre-metastatic niche, tumor cells home to the metastatic site then colonize and support vessel budding. OC: ovarian cancer; NK: natural killer; HUVECs: Human umbilical vein endothelial cells; TAM: tumor-associated macrophages; PMC: Peritoneal mesothelial cell; CAF: cancer-associated fibroblasts.

Overall, current works are investigating IL-6 receptor antagonists, classically used to treat rheumatoid arthritis, for their potential anti-cancer effects [82].

\section{Exosomes breach the barriers to tumor invasion within the pre-metastatic niche}

Approximately $70 \%$ of ovarian cancer patients have peritoneal metastasis at the time of surgery [83]. Typically, nests of tumor cells involve the peritoneum, omentum, mesentery, diaphragm, and surface of abdominopelvic viscera (Fig. 3a). These niduses of metastasis result from transport within the ascites; however, cases of hematogenous metastasis to the omentum also occur [84] (Fig. 3a). Histologically, both the omentum and peritoneum are loose collagen networks with vascularized connective tissue, scattered fibroblasts, adipocytes, and macrophages. They are covered by a single layer of microvilli-rich mesothelial cells and a thin basement membrane [85](Fig. 3b), which the circulating ovarian cancer cells must breach. The method of metastatic transport determines the barriers to invasion. In the hematogenous pathway, tumor cells penetrate surface endothelium, and, in the ascites pathway, they infiltrate the mesothelium (Fig. 3c).

Ovarian cancer exosomes advance angiogenesis by inducing vascular endothelial cell migration, which facilitates tumor cell homing to the pre-metastatic niche [58-60]. The notable absence of mesothelium in peritoneal viscera with metastatic growth suggests these cells are cleared during tumorigenesis, and possibly prior to large-scale metastatic invasion [86-88]. Mechanistically, ovarian cancer cells release exosomes with specific miRNAs (e.g miR-21 and miR-29a) into the ascites that remodel the mesothelial cell layer for enhanced peritoneal penetration [42] (Fig. 2e). Serum miR-99a-5p is significantly elevated in ovarian cancer patients and promotes cell invasion by affecting human peritoneal 
mesothelial cells (HPMCs) via fibronectin and vitronectin upregulation [43]. CD44 is overexpressed in the peritoneal mesothelial cells of ovarian cancer patients with omental metastasis. This occurs via an exosome conditioned pathway, whereby ovarian cancer exosomes transfer CD44 to peritoneal mesothelium causing its physical barrier to be cleared [37]. MMP1 expression is a negative factor of ovarian cancer prognosis. The mRNA of these proteases are horizontally transferred from ovarian cancer exosomes to peritoneal mesothelium, causing apoptosis of the peritoneal mesothelium through selfdestruction [44].

\section{Exosomes as pre-metastatic niche biomarkers and therapeutic applications}

Early detection of the pre-metastatic niche prior to metastasis remains an important goal within the field of gynecologic oncology, especially given the substantially worse outcomes associated with late stage diagnosis. Therefore, new techniques have sought to detect hyperpermeable, hypoxic, and inflammatory areas in addition to areas with altered extracellular matrix profiles characteristic of the metastatic niche. However, many of these techniques have low accuracy and are not yet suitable for clinical application [89-91]. Exosomes possess several unique advantages as biomarkers of pre-metastatic niche formation, as they are extremely stable, abundant, and tumor-specific. They are, therefore, promising biomarkers within the blood or ascites that warrant serious investigation in this deadly cancer, especially given their successes in other cancers [92]. For example, exosomes derived from pancreatic tumors bearing migration inhibitory factor (MIF) are selectively ingested by Kupffer cells within the liver, and therefore serve as important initiating factors in hepatic pre-metastatic niche formation [93]. Similarly, exosomal levels of the melanoma-specific protein tyrosinase-related protein 2 (TYRP2) has gained interest as a predictor of metastasis in melanoma [94].

Liquid biopsy is a technique where circulating tumor cells, cell-free nucleic acids, and tumor-derived exosomes can be analyzed from body fluids such as blood or ascites. The first exosome-based cancer diagnostic product was introduced to market within the United States on January 21, 2016 [95]. This technique is especially applicable to ovarian cancer diagnostics, as exosomes can be readily detected from ascites in a relatively non-invasive manner [96]. The most advanced biosensors in liquid biopsy detect cancerderived exosomes via highly specific target selection, biologic antigen sensing, and signal transduction techniques [97]. As to target selection, levels of exosomal miR-200b and miR-200c are associated with poor outcomes in ovarian cancer and significantly correlate with the ovarian tumor marker CA-125 [98]. Cancer-related antigens are prominent on the surface of exosomes and amenable to highly sensitive cancer cell detection. Examples include exosomes enriched with VEGF-A, semaphorin-3A, and TGF-beta in glioma (GBMs), HIF1- $\alpha$ in nasopharyngeal carcinoma, and MT1 MMP in fibrosarcoma and melanoma [22]. These proteins associated with exosomes are unique to their cancer of origin, and therefore promising targets of liquid biopsy detection especially for patients with an unknown cancer subtype. To this effect, various malignancies have been studied including melanoma, nasopharyngeal carcinoma, breast cancer, colorectal cancer, and ovarian cancer [97]. Very recently, a microfluidic chip-based liquid biopsy was able to isolate exosomes with detailed protein and signaling pathway profiles in ovarian cancer [99].

Therapeutic exosome-based strategies are also emerging, and function by exploiting the homing effect of exosomes on primary cancer cells [100]. A premetastatic niche mimic was generated by embedding ovarian cancer-exosomes into engineered biomaterials and implanted within the abdominal cavity of a murine model. This artificial pre-metastatic niche effectively recruited and trapped free ovarian cancer cells from the ascites, thus preventing these cells from homing towards normal pelvic viscera. Survival was substantially increased [101]. Another emerging technology utilizes celltargeting aptamer-modified extracellular vesicles with exosomes embedded in black phosphorus. While the aptamer directs the bioinspired extracellular vesicles towards targeted cells, the black phosphorous derived inorganic phosphate facilitates cell biomineralization [102]. This targeted technology has potential for elimination of the pre-metastatic niche and is a novel area of future chemotherapeutic research.

Tumor-derived exosomes undergo homing to preferred organ and cell-specific sites when preparing the pre-metastatic niche. Exosomal proteomics have revealed expression patterns of integrins to play a major role in this process. While the exosomal integrins $\alpha_{6} \beta_{4}$ and $\alpha_{6} \beta_{1}$ promote lung metastasis, $\alpha_{v} \beta_{5}$ is linked to liver metastasis. Therapeutically, targeting integrins $\alpha 6 \beta 4$ and $\alpha v \beta 5$ reduces exosome uptake and lung and liver metastasis, respectively [103]. These exosomal integrins are an additional predictor of pre-metastatic niche formation in liquid biopsy, and especially valuable for cancers with high rates of metastasis. Overall, because different cancers exhibit distinct mechanisms and exosome profiles in their respective premetastatic niches [93, 103, 104], it is likely that tumor specific biomarkers and therapeutic strategies will have acceptable specificity. In addition, given the premetastatic biomarker profile housed within ovarian cancer exosomes and their roles in pre-metastatic niche formation, recent advances in liquid biopsy diagnostics have made exosomes a promising new area of early screening and detection (Table 3 ). 


\section{Conclusions and perspectives}

Ovarian cancer exosomes promote pre-metastatic niche formation via immunosuppression, angiogenesis, stromal cell remodeling, and oncogenic reprogramming (Fig. 2). Since the pre-metastatic niche gained critical attention in 2005 [9], significant progress has been made in understanding the contribution of exosomes in conditioning the pre-metastatic niche for subsequent rapid metastatic growth. However, the roles of cell-shed exosomes have only recently been appreciated for their substantial effects on shaping the tumor microenvironment. In the preclinical work to date, exosomes in animal studies have been isolated and purified in vitro. Emerging technologies such as liquid biopsy will likely further characterize their tumorigenic effects in vivo, and may help to fully reveal the clinical significance of these pro-metastatic factors in ovarian cancer.

\section{Abbreviations}

ADSCs: Adipose tissue-derived mesenchymal stem cells; AKT: Protein kinase B; ALIX/PDCD6IP: programmed cell death 6-interacting protein; ARF6: ADPribosylation factor 6; ATF2: Activating transcription factor 2; COIA1: Collagen alpha-1 (XVIII) chain; DCs: Dendritic cells; ECs: Endothelial cells; EOC: Epithelial ovarian cancer; EOC: Epithelial ovarian cancer; EPHA2: Ephrin type-A receptor 2; ESCRT: Endosomal sorting complex required for transport; FasL: Fas ligand; GD 3: Ganglioside 3; GNA12: Guanine nucleotide-binding protein subunit alpha-12; HEK293: Human embryonic kidney; HIF: Hypoxia-inducible factor; HPMC: Human peritoneal mesothelial cells; HPMCs: Human peritoneal mesothelial cells; HUVECs: Human umbilical vein endothelial cells; HUVECs: Human umbilical vein endothelial cells; ILV: Intraluminal vesicle; LIN28: Lin-28 homolog A; LPA: Lysophosphatidic acid; MMP: Matrix metallopeptidase; MSCs: Mesenchymal stem cells; MTA1: Metastasisassociated protein 1; MVEs: Multivesicular endosomes; NK: Natural killer; OC: Ovarian cancer; PBMCs: Peripheral blood lymphocytes; PI3K: Phosphoinositide 3-kinases; PS: Phosphatidylserine; RISCs: Coiled-Coil Containing Protein Kinase; RNA: Induced silencing complexes; ROCK: Rho-Associated; SDF-1: Stromal cellderived factor 1; sE-cad: Soluble E-cadherin; SOCS: Suppress cytokine signaling; STAT3: Signal transducer and activator of transcription 3; TAMs: Tumor-associated macrophages; TCR: T-cell receptor; TLR: Toll-like receptor; TSG101: Tumor susceptibility gene 101 protein; VPS4: Vacuolar protein sorting-associated protein; a-SMA: Alpha-smooth muscle actin

\section{Acknowledgements}

We thank the first affiliated hospital of Zhengzhou University and the David Geffen School of Medicine at UCLA for their excellent technical assistance.

\section{Authors' contributions}

WLF was a major writer of the manuscript, and designed the figures, tables, researched appropriate references. ZFD and HRS developed the structure of the article and guided the selection of references. DC.D and FJ.H were responsible for language modification. All the authors read and approved the final manuscript.

\section{Funding}

WLF is supported by an overseas visiting scholarship from the Zhengzhou University of China. ZFD is supported, in part, through a Grant from Sarcoma Foundation of America (SFA) (222433), and a Grant from National Cancer Institute (NCI)/National Institutes of Health (NIH), UO1, CA151452-01.

\section{Availability of data and materials}

Not applicable.

Ethics approval and consent to participate Not applicable.

\section{Consent for publication}

All the authors have approved the final manuscript for publication.

\section{Competing interests}

The authors declare that they have no competing interests.

\section{Author details}

${ }^{1}$ Department of Obstetrics and Gynecology, The First Affiliated Hospital of Zhengzhou University, 1 Jianshe East Road, Zhengzhou 450052, Henan, China. ${ }^{2}$ Department of Orthopaedic Surgery, David Geffen School of Medicine at UCLA, 615 Charles, E. Young. Dr. South, Los Angeles, CA 90095, USA.

Received: 1 June 2019 Accepted: 24 July 2019

Published online: 13 August 2019

\section{References}

1. Howlader N NA, Krapcho M, Miller D, Bishop K, Kosary CL, Yu M, Ruhl J, Tatalovich Z, Mariotto A, Lewis DR, Chen HS, Feuer EJ, Cronin KA (eds). Statistics Review, 1975-2014, National Cancer Institute. 2018.

2. Torre LA, Trabert B, DeSantis CE, Miller KD, Samimi G, Runowicz CD, Gaudet MM, Jemal A, Siegel RL. Ovarian cancer statistics, 2018. CA Cancer J Clin. 2018;68:284-96

3. Jayson GC, Kohn EC, Kitchener HC, Ledermann JA. Ovarian cancer. Lancet. 2014;384:1376-88

4. Siegel RL, Miller KD, Jemal A. Cancer statistics, 2018. CA Cancer J Clin. 2018; 68:7-30.

5. Lee W, Ko SY, Mohamed MS, Kenny HA, Lengyel E, Naora H. Neutrophils facilitate ovarian cancer premetastatic niche formation in the omentum. J Exp Med. 2019;216:176-94.

6. Li K, Chen Y, Li A, Tan C, Liu X. Exosomes play roles in sequential processes of tumor metastasis. Int J Cancer. 2019;144:1486-95.

7. Mashouri L, Yousefi H, Aref AR, Ahadi AM, Molaei F, Alahari SK. Exosomes: composition, biogenesis, and mechanisms in cancer metastasis and drug resistance. Mol Cancer. 2019;18:75.

8. Yu Z, Zhao S, Ren L, Wang L, Chen Z, Hoffman RM, Zhou J. Pancreatic cancer-derived exosomes promote tumor metastasis and liver premetastatic niche formation. Oncotarget. 2017:8:63461-83.

9. Kaplan RN, Riba RD, Zacharoulis S, Bramley AH, Vincent L, Costa C, MacDonald DD, Jin DK, Shido K, Kerns SA, et al. VEGFR1-positive haematopoietic bone marrow progenitors initiate the pre-metastatic niche. Nature. 2005:438:820-7.

10. Psaila B, Lyden D. The metastatic niche: adapting the foreign soil. Nat Rev Cancer. 2009;9:285-93.

11. Melo SA, Sugimoto H, O'Connell JT, Kato N, Villanueva A, Vidal A, Qiu L, Vitkin E, Perelman LT, Melo CA, et al. Cancer exosomes perform cellindependent microRNA biogenesis and promote tumorigenesis. Cancer Cell. 2014;26:707-21.

12. Rivoltini L, Chiodoni C, Squarcina P, Tortoreto M, Villa A, Vergani B, Burdek M, Botti L, Arioli I, Cova A, et al. TNF-related apoptosis-inducing ligand (TRAIL)-armed exosomes deliver Proapoptotic signals to tumor site. Clin Cancer Res. 2016;22:3499-512.

13. Ye SB, Li ZL, Luo DH, Huang BJ, Chen YS, Zhang XS, Cui J, Zeng YX, Li J. Tumor-derived exosomes promote tumor progression and T-cell dysfunction through the regulation of enriched exosomal microRNAs in human nasopharyngeal carcinoma. Oncotarget. 2014;5:5439-52.

14. Au Yeung CL, Co NN, Tsuruga T, Yeung TL, Kwan SY, Leung CS, Li Y, Lu ES, Kwan K, Wong KK, et al. Exosomal transfer of stroma-derived miR21 confers paclitaxel resistance in ovarian cancer cells through targeting APAF1. Nat Commun. 2016;7:11150

15. Lobb RJ, Lima LG, Moller A. Exosomes: key mediators of metastasis and premetastatic niche formation. Semin Cell Dev Biol. 2017;67:3-10.

16. Guo Y, Ji X, Liu J, Fan D, Zhou Q, Chen C, Wang W, Wang G, Wang H, Yuan $W$, et al. Effects of exosomes on pre-metastatic niche formation in tumors. Mol Cancer. 2019;18:39.

17. Whiteside TL. Exosomes and tumor-mediated immune suppression. J Clin Invest. 2016;126:1216-23.

18. Tang MK, Wong AS. Exosomes: emerging biomarkers and targets for ovarian cancer. Cancer Lett. 2015;367:26-33. 
19. Dorayappan KDP, Wallbillich JJ, Cohn DE, Selvendiran K. The biological significance and clinical applications of exosomes in ovarian cancer. Gynecol Oncol. 2016;142:199-205.

20. Nawaz M, Fatima F, Nazarenko I, Ekstrom K, Murtaza I, Anees M, Sultan A, Neder L, Camussi G, Valadi H, et al. Extracellular vesicles in ovarian cancer: applications to tumor biology, immunotherapy and biomarker discovery. Expert Rev Proteomics. 2016;13:395-409.

21. van Niel G, D'Angelo G, Raposo G. Shedding light on the cell biology of extracellular vesicles. Nat Rev Mol Cell Biol. 2018;19:213-28.

22. Xu J, Liao K, Zhou W. Exosomes regulate the transformation of Cancer cells in Cancer stem cell homeostasis. Stem Cells Int. 2018;2018:4837370.

23. Hurley JH. ESCRT complexes and the biogenesis of multivesicular bodies. Curr Opin Cell Biol. 2008;20:4-11.

24. Pegtel DM, Gould SJ. Exosomes. Annu Rev Biochem. 2019;88:487-514.

25. Azmi AS, Bao B, Sarkar FH. Exosomes in cancer development, metastasis, and drug resistance: a comprehensive review. Cancer Metastasis Rev. 2013; 32:623-42.

26. Clancy JW, Zhang Y, Sheehan C, D'Souza-Schorey C. An ARF6-Exportin-5 axis delivers pre-miRNA cargo to tumour microvesicles. Nat Cell Biol. 2019;21: 856-66

27. Peng $P, Y a n Y$, Keng $S$. Exosomes in the ascites of ovarian cancer patients: origin and effects on anti-tumor immunity. Oncol Rep. 2011;25:749-62.

28. Runz S, Keller S, Rupp C, Stoeck A, Issa Y, Koensgen D, Mustea A, Sehouli J, Kristiansen G, Altevogt P. Malignant ascites-derived exosomes of ovarian carcinoma patients contain CD24 and EpCAM. Gynecol Oncol. 2007;107: 563-71

29. Taylor DD, Gercel-Taylor C. MicroRNA signatures of tumor-derived exosomes as diagnostic biomarkers of ovarian cancer. Gynecol Oncol. 2008;110:13-21.

30. Taylor DD, Gercel-Taylor C, Parker LP. Patient-derived tumor-reactive antibodies as diagnostic markers for ovarian cancer. Gynecol Oncol. 2009; 115:112-20.

31. Gong S, Li Q, Jeter CR, Fan Q, Tang DG, Liu B. Regulation of NANOG in cancer cells. Mol Carcinog. 2015;54:679-87.

32. Yamamoto CM, Oakes ML, Murakami T, Muto MG, Berkowitz RS, Ng SW Comparison of benign peritoneal fluid- and ovarian cancer ascites-derived extracellular vesicle RNA biomarkers. J Ovarian Res. 2018;11:20.

33. Sherman-Samis M, Onallah H, Holth A, Reich R, Davidson B. SOX2 and SOX9 are markers of clinically aggressive disease in metastatic high-grade serous carcinoma. Gynecol Oncol. 2019.153(3):651-660.

34. Liang B, Peng P, Chen S, Li L, Zhang M, Cao D, Yang J, Li H, Gui T, Li X, Shen K. Characterization and proteomic analysis of ovarian cancer-derived exosomes. J Proteome. 2013;80:171-82.

35. Yi H, Ye J, Yang X-M, Zhang L-W, Zhang Z-G, Chen Y-P. High-grade ovarian cancer secreting effective exosomes in tumor angiogenesis. Int J Clin Exp Pathol. 2015;8(5):5062-5070.

36. Sharma S, Alharbi M, Kobayashi M, Lai A, Guanzon D, Zuniga F, Ormazabal V, Palma C, Scholz-Romero K, Rice GE, et al. Proteomic analysis of exosomes reveals an association between cell invasiveness and exosomal bioactivity on endothelial and mesenchymal cell migration in vitro. Clin Sci (Lond). 2018;132:2029-44.

37. Nakamura K, Sawada K, Kinose Y, Yoshimura A, Toda A, Nakatsuka E, Hashimoto K, Mabuchi S, Morishige Kl, Kurachi H, et al. Exosomes promote ovarian Cancer cell invasion through transfer of CD44 to peritoneal mesothelial cells. Mol Cancer Res. 2017;15:78-92.

38. Enriquez VA, Cleys ER, Da Silveira JC, Spillman MA, Winger QA, Bouma GJ. High LIN28A expressing ovarian Cancer cells secrete exosomes that induce invasion and migration in HEK293 cells. Biomed Res Int. 2015;2015:701390.

39. Chen X, Ying X, Wang X, Wu X, Zhu Q, Wang X. Exosomes derived from hypoxic epithelial ovarian cancer deliver microRNA-940 to induce macrophage M2 polarization. Oncol Rep. 2017;38:522-8.

40. Ying X, Wu Q, Wu X, Zhu Q, Wang X, Jiang L, Chen X, Wang X. Epithelial ovarian cancer-secreted exosomal miR-222-3p induces polarization of tumor-associated macrophages. Oncotarget. 2016;7:43076-87.

41. Chen $X$, Zhou J, Li $X$, Wang $X$, Lin $Y$, Wang $X$. Exosomes derived from hypoxic epithelial ovarian cancer cells deliver microRNAs to macrophages and elicit a tumor-promoted phenotype. Cancer Lett. 2018;435:80-91.

42. Vaksman O, Trope C, Davidson B, Reich R. Exosome-derived miRNAs and ovarian carcinoma progression. Carcinogenesis. 2014;35:2113-20.

43. Yoshimura A, Sawada K, Nakamura K, Kinose Y, Nakatsuka E, Kobayashi M, Miyamoto M, Ishida K, Matsumoto $Y$, Kodama M, et al. Exosomal miR-99a-5p is elevated in sera of ovarian cancer patients and promotes cancer cell invasion by increasing fibronectin and vitronectin expression in neighboring peritoneal mesothelial cells. BMC Cancer. 2018;18:1065.

44. Yokoi A, Yoshioka Y, Yamamoto Y, Ishikawa M, Ikeda SI, Kato T, Kiyono T, Takeshita F, Kajiyama H, Kikkawa F, Ochiya T. Malignant extracellular vesicles carrying MMP1 mRNA facilitate peritoneal dissemination in ovarian cancer. Nat Commun. 2017;8:14470.

45. Kobayashi M, Salomon C, Tapia J, Illanes SE, Mitchell MD, Rice GE. Ovarian cancer cell invasiveness is associated with discordant exosomal sequestration of Let-7 miRNA and miR-200. J Transl Med. 2014;12:4.

46. Shenoy GN, Loyall J, Berenson CS, Kelleher RJ Jr, lyer V, Balu-lyer SV, Odunsi K, Bankert RB. Sialic acid-dependent inhibition of T cells by Exosomal ganglioside GD3 in ovarian tumor microenvironments. J Immunol. 2018;201: 3750-8

47. Bretz NP, Ridinger J, Rupp AK, Rimbach K, Keller S, Rupp C, Marme F, Umansky L, Umansky V, Eigenbrod T, et al. Body fluid exosomes promote secretion of inflammatory cytokines in monocytic cells via toll-like receptor signaling. J Biol Chem. 2013;288:36691-702.

48. Li Y, Yang Y, Xiong A, Wu X, Xie J, Han S, Zhao S. Comparative gene expression analysis of lymphocytes treated with exosomes derived from ovarian Cancer and ovarian cysts. Front Immunol. 2017;8:607.

49. Vesely MD, Schreiber RD. Cancer immunoediting: antigens, mechanisms, and implications to cancer immunotherapy. Ann N Y Acad Sci. 2013;1284:1-5.

50. Aguado BA, Bushnell GG, Rao SS, Jeruss JS, Shea LD. Engineering the premetastatic niche. Nat Biomed Eng. 2017;1:0077. https://doi.org/10.1038/ s41551-017-0077.

51. Filipazzi P, Burdek M, Villa A, Rivoltini L, Huber V. Recent advances on the role of tumor exosomes in immunosuppression and disease progression. Semin Cancer Biol. 2012:22:342-9.

52. Liu Y, Cao X. Characteristics and significance of the pre-metastatic niche Cancer Cell. 2016;30:668-81.

53. Taylor DD, Gercel-Taylor C, Lyons KS, Stanson J, Whiteside TL. T-cell apoptosis and suppression of T-cell receptor/CD3-zeta by Fas ligandcontaining membrane vesicles shed from ovarian tumors. Clin Cancer Res. 2003;9:5113-9.

54. Taylor DD, Gercel-Taylor C. Tumour-derived exosomes and their role in cancer-associated T-cell signalling defects. Br J Cancer. 2005;92:305-11.

55. Meng Y, Kang S, Fishman DA. Lysophosphatidic acid stimulates fas ligand microvesicle release from ovarian cancer cells. Cancer Immunol Immunother. 2005;54:807-14.

56. Kelleher RJ Jr, Balu-lyer S, Loyall J, Sacca AJ, Shenoy GN, Peng P, lyer V, Fathallah AM, Berenson CS, Wallace PK, et al. Extracellular vesicles present in human ovarian tumor microenvironments induce a phosphatidylserinedependent arrest in the T-cell signaling Cascade. Cancer Immunol Res. 2015:3:1269-78.

57. Labani-Motlagh A, Israelsson P, Ottander U, Lundin E, Nagaev I, Nagaeva O, Dehlin E, Baranov V, Mincheva-Nilsson L. Differential expression of ligands for NKG2D and DNAM-1 receptors by epithelial ovarian cancer-derived exosomes and its influence on NK cell cytotoxicity. Tumour Biol. 2016;37:5455-66.

58. Yi H, Ye J, Yang XM, Zhang LW, Zhang ZG, Chen YP. High-grade ovarian cancer secreting effective exosomes in tumor angiogenesis. Int J Clin Exp Pathol. 2015;8:5062-70.

59. Millimaggi D, Mari M, D'Ascenzo S, Carosa E, Jannini EA, Zucker S, Carta G, Pavan A, Dolo V. Tumor vesicle-associated CD147 modulates the angiogenic capability of endothelial cells. Neoplasia. 2007;9:349-57.

60. Tang MKS, Yue PYK, Ip PP, Huang RL, Lai HC, Cheung ANY, Tse KY, Ngan HYS, Wong AST. Soluble E-cadherin promotes tumor angiogenesis and localizes to exosome surface. Nat Commun. 2018;9:2270.

61. Ishii G, Ochiai A, Neri S. Phenotypic and functional heterogeneity of cancerassociated fibroblast within the tumor microenvironment. Adv Drug Deliv Rev. 2016;99:186-96.

62. Sleeman JP. The metastatic niche and stromal progression. Cancer Metastasis Rev. 2012;31:429-40.

63. Schauer IG, Sood AK, Mok S, Liu J. Cancer-associated fibroblasts and their putative role in potentiating the initiation and development of epithelial ovarian cancer. Neoplasia. 2011;13:393-405.

64. Yamamura $Y$, Asai N, Enomoto A, Kato T, Mii S, Kondo Y, Ushida K, Niimi K Tsunoda N, Nagino M, et al. Akt-Girdin signaling in cancer-associated fibroblasts contributes to tumor progression. Cancer Res. 2015;75:813-23.

65. Mitra AK, Zillhardt M, Hua Y, Tiwari P, Murmann AE, Peter ME, Lengyel E. MicroRNAs reprogram normal fibroblasts into cancer-associated fibroblasts in ovarian cancer. Cancer Discov. 2012;2:1100-8. 
66. Giusti I, Di Francesco M, D'Ascenzo S, Palmerini MG, Macchiarelli G, Carta G, Dolo V. Ovarian cancer-derived extracellular vesicles affect normal human fibroblast behavior. Cancer Biol Ther. 2018;19:722-34.

67. Cho JA, Park H, Lim EH, Kim KH, Choi JS, Lee JH, Shin JW, Lee KW. Exosomes from ovarian cancer cells induce adipose tissue-derived mesenchymal stem cells to acquire the physical and functional characteristics of tumorsupporting myofibroblasts. Gynecol Oncol. 2011;123:379-86.

68. Pollard JW. Trophic macrophages in development and disease. Nat Rev Immunol. 2009;9:259-70.

69. Mantovani A, Sozzani S, Locati M, Allavena P, Sica A. Macrophage polarization: tumor-associated macrophages as a paradigm for polarized M2 mononuclear phagocytes. Trends Immunol. 2002;23:549-55.

70. Sica A, Larghi P, Mancino A, Rubino L, Porta C, Totaro MG, Rimoldi M, Biswas SK, Allavena P, Mantovani A. Macrophage polarization in tumour progression. Semin Cancer Biol. Elsevier; 2008;(5):349-55.

71. Chen XW, Yu TJ, Zhang J, Li Y, Chen HL, Yang GF, Yu W, Liu YZ, Liu XX, Duan CF, et al. CYP4A in tumor-associated macrophages promotes premetastatic niche formation and metastasis. Oncogene. 2017;36:5045-57.

72. Kanlikilicer P, Bayraktar R, Denizli M, Rashed MH, Ivan C, Aslan B, Mitra R, Karagoz K, Bayraktar E, Zhang X, et al. Exosomal miRNA confers chemo resistance via targeting Cav1/p-gp/M2-type macrophage axis in ovarian cancer. EBioMedicine. 2018;38:100-12.

73. Yin M, Li X, Tan S, Zhou HJ, Ji W, Bellone S, Xu X, Zhang H, Santin AD, Lou $G$, Min W. Tumor-associated macrophages drive spheroid formation during early transcoelomic metastasis of ovarian cancer. J Clin Invest. 2016;126: 4157-73.

74. Li W, Zhang X, Wang J, Li M, Cao C, Tan J, Ma D, Gao Q. TGFbeta1 in fibroblasts-derived exosomes promotes epithelial-mesenchymal transition of ovarian cancer cells. Oncotarget. 2017:8:96035-47.

75. Glentis A, Oertle P, Mariani P, Chikina A, El Marjou F, Attieh Y, Zaccarini F, Lae M, Loew D, Dingli F, et al. Cancer-associated fibroblasts induce metalloprotease-independent cancer cell invasion of the basement membrane. Nat Commun. 2017:8:924.

76. Keklikoglou I, Cianciaruso C, Guc E, Squadrito ML, Spring LM, Tazzyman S, Lambein L, Poissonnier A, Ferraro GB, Baer C, et al. Chemotherapy elicits pro-metastatic extracellular vesicles in breast cancer models. Nat Cell Biol. 2019; 21(2):190-202.

77. Xu S, Yang Z, Jin P, Yang X, Li X, Wei X, Wang Y, Long S, Zhang T, Chen G, et al. Metformin suppresses tumor progression by inactivating stromal fibroblasts in ovarian Cancer. Mol Cancer Ther. 2018;17:1291-302.

78. Duan Z, Feller AJ, Penson RT, Chabner BA, Seiden MV. Discovery of differentially expressed genes associated with paclitaxel resistance using cDNA array technology: analysis of interleukin (IL) 6, IL-8, and monocyte chemotactic protein 1 in the paclitaxel-resistant phenotype. Clin Cancer Res. 1999:5:3445-53.

79. Duan Z, Foster R, Bell DA, Mahoney J, Wolak K, Vaidya A, Hampel C, Lee H, Seiden MV. Signal transducers and activators of transcription 3 pathway activation in drug-resistant ovarian cancer. Clin Cancer Res. 2006;12:5055-63.

80. Penson RT, Kronish K, Duan Z, Feller AJ, Stark P, Cook SE, Duska LR, Fuller AF, Goodman AK, Nikrui N, et al. Cytokines IL-1beta, IL-2, IL-6, IL-8, MCP-1, GM-CSF and TNFalpha in patients with epithelial ovarian cancer and their relationship to treatment with paclitaxel. Int J Gynecol Cancer. 2000;10:33-41.

81. Guo Y, Nemeth J, O'Brien C, Susa M, Liu X, Zhang Z, Choy E, Mankin H, Hornicek F, Duan Z. Effects of siltuximab on the IL-6-induced signaling pathway in ovarian cancer. Clin Cancer Res. 2010;16:5759-69.

82. Yoshida GJ, Azuma A, Miura Y, Orimo A. Activated fibroblast program orchestrates tumor initiation and progression; molecular mechanisms and the associated therapeutic strategies. Int J Mol Sci. 2019;20.

83. Tan DS, Agarwal R, Kaye SB. Mechanisms of transcoelomic metastasis in ovarian cancer. Lancet Oncol. 2006;7:925-34.

84. Pradeep S, Kim SW, Wu SY, Nishimura M, Chaluvally-Raghavan P, Miyake T, Pecot CV, Kim SJ, Choi HJ, Bischoff FZ, et al. Hematogenous metastasis of ovarian cancer: rethinking mode of spread. Cancer Cell. 2014;26:77-91.

85. Brady M, Mahoney E. In: Doherty GM, editor. Peritoneal cavity. In CURRENT Diagnosis \&amp; Treatment: Surgery, 14e. McGraw-Hill Education: New York; 2015.

86. Kenny HA, Nieman KM, Mitra AK, Lengyel E. The first line of intra-abdominal metastatic attack: breaching the mesothelial cell layer. Cancer Discov. 2011; $1: 100-2$.

87. Davidowitz RA, Selfors LM, Iwanicki MP, Elias KM, Karst A, Piao H, Ince TA, Drage MG, Dering J, Konecny GE, et al. Mesenchymal gene program- expressing ovarian cancer spheroids exhibit enhanced mesothelial clearance. J Clin Invest. 2014;124:2611-25.

88. Iwanicki MP, Davidowitz RA, Ng MR, Besser A, Muranen T, Merritt M, Danuser G, Ince TA, Brugge JS. Ovarian cancer spheroids use myosingenerated force to clear the mesothelium. Cancer Discov. 2011;1:144-57.

89. Shokeen M, Zheleznyak A, Wilson JM, Jiang M, Liu R, Ferdani R, Lam KS, Schwarz JK, Anderson CJ. Molecular imaging of very late antigen-4 (alpha4beta1 integrin) in the premetastatic niche. J Nucl Med. 2012;53:779-86.

90. Soodgupta D, Hurchla MA, Jiang M, Zheleznyak A, Weilbaecher KN, Anderson CJ, Tomasson MH, Shokeen M. Very late antigen-4 (alpha(4)beta(1) integrin) targeted PET imaging of multiple myeloma. PLoS One. 2013;8:e55841.

91. Hu L, Wickline SA, Hood JL. Magnetic resonance imaging of melanoma exosomes in lymph nodes. Magn Reson Med. 2015;74:266-71.

92. Kalluri R. The biology and function of exosomes in cancer. J Clin Invest. 2016;126:1208-15.

93. Costa-Silva B, Aiello NM, Ocean AJ, Singh S, Zhang H, Thakur BK, Becker A, Hoshino A, Mark MT, Molina H, et al. Pancreatic cancer exosomes initiate pre-metastatic niche formation in the liver. Nat Cell Biol. 2015;17:816-26.

94. Peinado H, Aleckovic M, Lavotshkin S, Matei I, Costa-Silva B, Moreno-Bueno G, Hergueta-Redondo M, Williams C, Garcia-Santos G, Ghajar C, et al. Melanoma exosomes educate bone marrow progenitor cells toward a prometastatic phenotype through MET. Nat Med. 2012;18:883-91.

95. Sheridan C. Exosome cancer diagnostic reaches market. Nat Biotechnol. 2016:34:359-60.

96. Andre F, Schartz NE, Movassagh M, Flament C, Pautier P, Morice P, Pomel C, Lhomme C, Escudier B, Le Chevalier T, et al. Malignant effusions and immunogenic tumour-derived exosomes. Lancet. 2002;360:295-305.

97. Cheng N, Du D, Wang X, Liu D, Xu W, Luo Y, Lin Y. Recent advances in biosensors for detecting Cancer-derived exosomes. Trends Biotechnol. 2019. https://doi.org/10.1016/j.tibtech.2019.04.008. [Epub ahead of print]

98. Meng X, Muller V, Milde-Langosch K, Trillsch F, Pantel K, Schwarzenbach H. Diagnostic and prognostic relevance of circulating exosomal miR-373, miR200a, miR-200b and miR-200c in patients with epithelial ovarian cancer. Oncotarget. 2016;7:16923-35.

99. Dorayappan KDP, Gardner ML, Hisey CL, Zingarelli RA, Smith BQ, Lightfoot MDS, Gogna R, Flannery MM, Hays J, Hansford DJ, et al. A microfluidic Chip enables isolation of exosomes and establishment of their protein profiles and associated signaling pathways in ovarian Cancer. Cancer Res. 2019;79: 3503-13.

100. Wu M, Wang G, Hu W, Yao Y, Yu XF. Emerging roles and therapeutic value of exosomes in cancer metastasis. Mol Cancer. 2019;18:53.

101. de la Fuente A, Alonso-Alconada L, Costa C, Cueva J, Garcia-Caballero T, Lopez-Lopez R, Abal M. M-trap: exosome-based capture of tumor cells as a new technology in peritoneal metastasis. J Natl Cancer Inst. 2015;107(9). https://doi.org/10.1093/jnci/djv184.

102. Wang Y, Hu X, Zhang L, Zhu C, Wang J, Li Y, Wang Y, Wang C, Zhang Y, Yuan Q. Bioinspired extracellular vesicles embedded with black phosphorus for molecular recognition-guided biomineralization. Nat Commun. 2019;10:2829.

103. Hoshino A, Costa-Silva B, Shen TL, Rodrigues G, Hashimoto A, Tesic Mark M, Molina H, Kohsaka S, Di Giannatale A, Ceder S, et al. Tumour exosome integrins determine organotropic metastasis. Nature. 2015;527:329-35.

104. Peinado H, Alečković M, Lavotshkin S, Matei I, Costa-Silva B, Moreno-Bueno G, Hergueta-Redondo M, Williams C, García-Santos G, Ghajar CM. Melanoma exosomes educate bone marrow progenitor cells toward a pro-metastatic phenotype through MET. Nat Med. 2012;18:883.

\section{Publisher's Note}

Springer Nature remains neutral with regard to jurisdictional claims in published maps and institutional affiliations. 